\title{
Fra spiritualità e materialismo. Sulle traduzioni settecentesche dell'"Inferno" dantesco
}

\section{Laura Brignoli}

\section{Q OpenEdition}

1 Journals

\section{Edizione digitale}

URL: https://journals.openedition.org/studifrancesi/9741

DOI: 10.4000/studifrancesi.9741

ISSN: 2421-5856

\section{Editore}

Rosenberg \& Sellier

\section{Edizione cartacea}

Data di pubblicazione: 1 octobre 2007

Paginazione: 378-389

ISSN: 0039-2944

\section{Notizia bibliografica digitale}

Laura Brignoli, «Fra spiritualità e materialismo. Sulle traduzioni settecentesche dell "Inferno" dantesco», Studi Francesi [Online], 152 (LI | II) | 2007, online dal 30 novembre 2015, consultato il 20 novembre 2021. URL: http://journals.openedition.org/studifrancesi/9741 ; DOI: https://doi.org/ 10.4000/studifrancesi.9741

\section{(c) (i) (9)}

Studi Francesi è distribuita con Licenza Creative Commons Attribuzione - Non commerciale - Non opere derivate 4.0 Internazionale. 


\title{
Fra spiritualità e materialismo. Sulle traduzioni settecentesche dell' 'Inferno' dantesco
}

\begin{abstract}
Hubert remarqua combien Dante était difficile à vêtir d'un convenable vêtement étranger. Il pardonna aux hommes de bonne volonté, qui l'avaient tenté, leurs scandaleuses traductions: on ne pouvait peut-être faire mieux qu'en adoptant une exacte barbarie aussi défigurante que les métaphores: la précision de l'original devient de la sécheresse; sa clarté, de la pénombre, car il faut employer certains mots courts dont le sens vrai s'est perdu et d'autres qui ne se lisent plus que dans les glossaires. Finalement, il se posa cet aphorisme: on ne peut pas traduire en une langue vieille et raffinée une oeuvre appartenant à la jeunesse d'une langue consanguine.
\end{abstract}

(Rémy de Gourmont, Sixtine)

Un'opera come la Commedia segna, anche sul versante letterario, l'inizio della decadenza del latino come lingua universale. Dante infatti sceglie per la sua summa antropologica una lingua giovane come il fiorentino, non ancora consacrata dall'uso letterario. Che il poeta fosse conscio della necessità di nobilitare la lingua è evidente dall'invito che egli rivolge, nella Vita Nuova, ai rimatori volgari affinché imitino i poeti classici della latinità, come fa lui stesso nella scelta degli argomenti capaci di conferire dignità a una lingua che non ne aveva ancora. In questo caso, dunque, giovinezza della lingua corrisponde alla mancanza di quella lunga tradizione letteraria atta a consacrarne la nobiltà, che il fiorentino non possedeva ancora, senza per questo essere privo di lustro: $\mathrm{i}$ «fondamenti grammaticali e lessicali» della sua lingua sono «senza alcun dubbio fiorentini, egli si vale liberamente di tutte le risorse linguistiche che abbiano già avuto una consacrazione letteraria» ${ }^{1}$. Sappiamo che Dante di fatto attua una mescolanza linguistica straordinaria non certo destinata a piacere a quei letterati di Francia che, nel lungo periodo storico in cui si andava affermando l'ideale classico, sostenevano la necessità di una separazione dei livelli stilistici. Per essi, la ricerca stilistica dantesca, ricca di espressioni colorite, appariva come una caduta nella più bassa trivialità: non è un caso che il Seicento francese abbia praticamente ignorato il capolavoro di Dante.

La descrizione di un simbolico viaggio nell'oltretomba poteva invece cominciare ad affascinare i letterati tardo settecenteschi, per quanto essi stessi, impregnati di una lingua classica spogliata di ogni audacia sia stilistica che lessicale, potessero ritrarsi di fronte alla sua traboccante ricchezza linguistica².

La ragione per la quale, in Francia e non solo, si comincia a guardare oltre la grossolanità linguistica della Commedia è principalmente da addurre, come abbiamo già rilevato ${ }^{3}$, in un mutamento del gusto che induceva ad apprezzare i versi aspri e

(1) B. Migliorini, Storia della lingua italiana, Firenze, 1960, p. 188.

(2) A. FARINELli, nel suo Dante e la Francia (Milano, Hoepli, 1908, vol. II, p. 251), sostiene con ragione l" indifferenza "pressoché assoluta" nel primo Settecento per Dante in Francia.
(3) Si veda la nostra comunicazione presentata al Convegno "La nascita del concetto di traduzione nelle nazioni europee fra enciclopedismo ed epoca romantica", Milano, 18-20 novembre 1999, Armando editore, 2001, pp. 228-242. 
selvaggi, ma carichi di pathos, del divino poeta. Sappiamo che un'accentuata sensibilità preromantica è ciò che induce Rivarol, pur mantenendosi nei limiti stretti dello stile sublime, a enfatizzare i chiaroscuri e a insistere sulle tenebre della dimora infernale. Ma questo può non essere l'unico motivo che ha acceso l'interesse. Prima di lui, infatti, il conte Colbert d'Estouteville (o Détouteville) e Moutonnet de Clairfons traducono la Divina Commedia con risultati diametralmente opposti essendo, verosimilmente, incuriositi da altri aspetti.

Sarà dunque interessante mettere a confronto queste due ultime traduzioni, senza precluderci qualche episodico rinvio a Rivarol di cui ci siamo occupati in precedenza. Lo scopo non è certo quello di stabilire quale sia la migliore, valutazione in qualche modo spontanea ma inevitabilmente sottomessa al gusto odierno, e come tale esposta a variabili. Cercheremo piuttosto di mettere in luce quali spinte stiano alla base dell'una e dell'altra, quali aspetti del capolavoro dantesco abbiano sollecitato i suoi traduttori.

Si è ritenuto non solo inevitabile, ma addirittura opportuno l'inserimento di molte citazioni: la diffusione di opere difficilmente reperibili non sarà meno importante del fornire prove alle nostre affermazioni.

\section{Confronti}

L'assunzione di un inevitabile per quanto scontato principio cronologico induce a scegliere quale punto di partenza la traduzione di Détouteville. Ė vero che l'editore Sallior la pubblica solo nel 1796, dopo averla integrata delle parti mancanti e aver rettificato alcuni passaggi evidentemente scorretti. Ma la presenta in prefazione come la prima traduzione della Divina Commedia in prosa: se era rimasta fino ad allora inedita, non fu sconosciuta, tanto è vero che Montesquieu ne raccomandava la lettura all'abate Guasco e che Moutonnet de Clairfons ne criticava aspramente le scelte traduttive. Sallior non fornisce alcuna data di composizione. L'Enciclopedia Dantesca la colloca nel 1751.

L'editore era senza dubbio cosciente delle imperfezioni di questa traduzione che, con involontario eufemismo, descrive come non priva di difetti ${ }^{4}$, ma vi rileva il non debole merito di essere l'unica traduzione disponibile delle tre cantiche, sia Rivarol che Moutonnet essendosi infatti limitati alla prima parte del poema. Insomma Sallior constata l'impellente necessità di far conoscere il poema nella sua interezza e questa traduzione deve servire a «donner au public une première idée de l'ensemble d'un poème très singulier et qui présente de très grandes beautés. Cette traduction est, pour ainsi dire, la préface d'une traduction plus digne de l'original» ${ }^{5}$. Il suo zelo di editore lo induce comunque a presentarla come l'unica a non offrire una visione distorta del poema, come era accaduto a suo dire negli altri casi: le difficoltà commerciali incontrate dalla traduzione di Moutonnet sono addotte a prova della sua mediocrità, attribuendo indirettamente al pubblico una capacità di giudizio che lascia perplessi ${ }^{6}$. Certo la richiesta di un testo accessibile al pubblico francese doveva essere

(4) «cette traduction [...] sans doute n'est pas sans défauts», La Divine Comédie de Dante Alighieri, traduite par Détouteville, Paris, chez Sallior, 1796, Préface, p. VI.

(5) Ibid.

(6) «Depuis vingt ans, le public n'a pas encore épuisé l'édition, et cependant plusieurs libraires ont employé les moyens usités pour tirer ce livre de sa léthargie commerciale. On peut donc affirmer que le travail de Moutonnet, bien loin d'être utile à la réputation de Dante, en a retardé de beaucoup le progrès. En 1785, Rivarol donne une nouvelle traduction de l'Enfer. Elle eut plus de succès que la précédente; elle le méritoit à quelques égards. Elle est mieux écrite en Français [...]». Ibid., Préface, p. III. 
abbastanza importante, anche se verosimilmente esiguo era il numero di quanti erano in grado di giudicare, come fa Sallior, la traduzione di Rivarol eccessivamente raffinata rispetto all'originale: non a caso lo accusa di trasformare il "genio" robusto di Dante in leggiadro "esprit". Sallior, a differenza di Rivarol, non era convinto dell'eccellenza del francese e considerava negativamente le imposizioni della lingua classica il cui unico effetto consisteva nel ridurre i colori dell'espressione ${ }^{7}$.

A Détouteville va dunque riconosciuto il merito di aver riempito un vuoto che durerà fino all'inizio del XIX secolo, quando finalmente la traduzione della Divina Commedia verrà pubblicata integralmente ${ }^{8}$. È una versione del tutto priva di note esplicative (per approfondire un eventuale interesse per le vicende storiche evocate, Sallior rimanda, nella prefazione, alle due traduzioni che lo hanno preceduto); tipograficamente, non presenta capoversi. Probabilmente nel tentativo di rappresentare fedelmente il susseguirsi costante delle terzine, Sallior riporta un solo paragrafo per ciascun canto, ma l'effetto mimetico è tutt'altro che raggiunto: l'agilità delle terzine non può essere riprodotta con un continuum in prosa che genera solo lo spiacevole risultato di rendere più faticosa la lettura.

L'intento primitivo di Moutonnet de Clairfons si delinea come una reazione a Détouteville, la cui traduzione, come si è detto, circolava in forma manoscritta. Ammiratore entusiasta di Dante, voleva offrire al pubblico una versione più adeguata dell'opera dantesca rispetto alla piatta traduzione del suo predecessore e, nel 1776, comincia a dare alle stampe l'Inferno con l'intenzione di pubblicare in seguito le altre due cantiche: «Si l'on paroît content de cette Traduction, je ferai imprimer dans la suite, celle du Purgatoire \& du Paradis. J'ai voulu d'abord sonder le goût du Public par l'Enfer»'. Questa affermazione ha indotto a credere che Moutonnet avesse tradotto interamente il poema dantesco. Tuttavia non si è mai trovata traccia né del Purgatorio né del Paradiso. La sua traduzione non ebbe la diffusione che l'autore si era atteso ed è stato ipotizzato che fosse stata proprio questa la ragione che lo indusse ad abbandonare l'impresa.

Nella sua prefazione, intitolata "Vie de Dante", Moutonnet si preoccupa di fornire giudizi critici sul poema, e la sua sensibilità filologica è provata dalla menzione precisa delle fonti da cui ha tratto le sue citazioni; ciascuno sa quanto sia raro un tale atteggiamento.

Per questo non si può che essere sorpresi nel constatare il suo dichiarato disinteresse per la precisione della costruzione dantesca, al punto che nella prefazione (pp. 36-37), giudica vano e puerile verificare le misure dell'Inferno e dei suoi abitanti (ad esempio i giganti). È forse da attribuire a questa indifferenza la sua contraddittoria definizione del cono infernale a cerchi concentrici ma degradante «en forme de spirale» (p. 38).

Fra i meriti della sua traduzione, bisogna segnalare la pubblicazione del testo originale a fronte della sua versione in $\operatorname{prosa}^{10}$ : atto coraggioso che espone il suo lavo-

(7) Ibid., Préface, p. II.

(8) La prima traduzione completa della Divina Commedia risale agli anni 1811-13, ed è dovuta ad Artaud de Montor.

(9) La Divine Comédie de Dante Alighieri, par Moutonnet de Clairfons, Paris, Leclerc, Le Boucher, 1776, Préface, p. 45

(10) L'unica opera consultabile alla Bibliothèque Nationale di Parigi è la seguente: LA / DIVINE COMÉDIE / DE / DANTE ALIGHIERI, / L'ENFER; / TRADUCTION FRANÇOISE. / Ac- compagnée du texte, de Notes historiques, critiques, / E de la Vie du Poète. / PAR M. Moutonnet DE Clairfons./ O voi, ch'avete gl'intelletti sani, / Mirate la Dottrina, che s'asconde / Sotto 'l velame degli Versi strani! / Dante, Inf. Cant. IX, Terz. 21 / A Florence / Et se trowve à Paris, / Chez LE CLERC, LE BOUCHER, Libraires, Quai des Argentins. / M. DCC. LXXVVI. Ciò non toglie che ne esista un'altra, con segnatura diversa, ma pubblicata sempre a Firenze e a Parigi, della quale il catalogo fornisce notizie incomplete. 
ro al giudizio immediato, ma che non può essere attribuito al Moutonnet medesimo: se l'iniziativa fosse sua, infatti, non avrebbe avuto motivo di soffermarsi sui passi più complessi per il traduttore, fornendo in nota i versi dell'originale oltre alle diverse possibili traduzioni ${ }^{11}$.

Moutonnet è senza dubbio più colto di Rivarol e Détouteville. Nelle note di cui correda la sua traduzione cita spesso passaggi delle Sacre Scritture o di poeti latini senza tradurli; sensibile al suono della lingua, li considera «comme des diamans d'une belle eau, parfaitement taillés, \& qui perdroient trop à être retouchés» (p. 59); mette a confronto delle metafore giudicate troppo ardite per il gusto francese con brani di poemi coevi, italiani o francesi, e giunge persino a indignarsi che taluni critici, certamente incapaci di emulare tanta grandezza, osino biasimare le scelte poetiche di Dante (p. 62).

Non bisogna ricercare in queste prime traduzioni quella ricchezza stilistica che caratterizza l'originale. Ciò che risulta irrimediabilmente riduttivo, è la loro sostanziale impossibilità di riprodurre, con lo stesso intento enciclopedico di Dante, quella variazione degli stili e quel repertorio idiomatico peculiari alla Commedia. La mancanza di una lingua duttile ne è la ragione principale.

Tuttavia Moutonnet ci fornisce una traduzione di buon livello stilistico, pur nel rispetto dei canoni classicistici. Non lo stesso si può dire per Détouteville, che mira a trasporre grosso modo il significato globale, senza nessun tipo di sensibilità stilistica né di virtuosismo verbale. Un abisso separa le due traduzioni e Moutonnet puntava proprio alla massima distanza dal suo predecessore. Persino laddove potrebbe sembrare che Détouteville avesse colto l'intento stilistico di Dante, la sua resa è poeticamente inferiore. Ne è un esempio il passo nel quale Dante, descrivendo la selva dei suicidi, rende più incisiva la sua antitesi grazie alla struttura simmetrica dei versi:

Non fronda verde, ma di color fosco; non rami schietti, ma nodosi e involti:

non pomi v'eran, ma stecchi con tosco (XIII, 4-6).

\section{Détouteville rispetta l'antitesi:}

Les feuilles n'y étoient point vertes, mais brunes. Les branches n'étoient point unies, mais crochues et pleines de noeuds. Les arbres ne portoient point de fruits, mais étoient hérissés d'épines empoisonnées.

Ma questa traduzione risulta alla fine monotona se confrontata sia a Rivarol che, inserendo una metafora, anticipa l'ambigua natura di quei vegetali:

des troncs sans verdures et sans fruits, couverts de feuilles noirâtres, étendaient leurs bras tortueux, hérissés de noeuds difformes et d'épines empoisonnées.

sia a Moutonnet, che sostituisce alla simmetria dantesca una gradazione sintattica idonea a rinforzare quella semantica:

(11) Eccone qualche esempio: p. 79: «Pour désigner la Lune, il se sert de cette périphrase: Le Ciel qui a les plus petits cercles: da quel Ciel, c'i ha minor le cerchi suoi». p. 95: «Leur crainte se change alors en désir. Tel est le sens du Vers Italien: Si che la tema si volge in disio»; pp.108-109: «Le Texte Italien peut offrir un autre sens, \& l'on devroit alors traduire ainsi: la Poësie épique est la plus sublime: semblable à l'Aigle, elle l'emporte sur tous les autres Poëmes.

Di quel Signor dell'altissimo canto,

Che sovra gli altri, com'aquila, vola».

p. 175: «On peut rendre différemment cet endroit: Quivi'l lasciammo, che più non ne narro: Laissons-le; je ne pourrois rien dire de plus fort» ecc. 
Les feuilles des arbres n'étoient pas vertes, mais brunes; ni les branches droites, mais toutes noueuses \& tortues: au lieu de fruits, l'on ne trouvoit que des épines empoisonnées.

Comunque, la severità del giudizio che Moutonnet formula sulla traduzione manoscritta di Détouteville non gli impedisce di farne abbondante uso, come si vede dagli esempi riportati qui di seguito:

\section{DANTE}

Pape Satan, pape Satan

aleppe,

Cominciò Pluto, con la voce

chioccia:

E quel savio gentil, che tutto

seppe

Disse, per confortarmi: Non

ti noccia

La tua paura, che poder,

ch'egli abbia,

Non terrà lo scender questa

roccia

(Inf. VII, vv. 1-6)

Da tutte parti l'alta valle feda

Tremò sì , ch'i' pensai che

l'universo

Sentisse amor, per lo qual è,

chi creda

Più volte il mondo in caosso

converso:

(Inf. XII, vv. 40-43)

\section{DÉTOUTEVILLE}

Dès que je parus, ce monarque infernal se mit à crier d'une voix enrouée: Satan, Satan, alerte, alerte. Alors Virgile toujours sage me dit pour me rassurer: ne vous laissez point abattre par la peur. Quelque puissance que Pluton puisse avoir, il ne nous empêchera pas de pénétrer plus avant.

cette vallée trembla tellement, qu'on crut que l'univers entier alloit rentrer dans le cahos (sic).

\section{MOUTONNET}

Ho Satan! ho Satan! ah! s'écrie Pluton d'une voix enrouée. Mon Guide généreux \& instruit de tout, me dit alors, pour m'encourager: ne vous laissez point abattre par la crainte; quel que soit son pouvoir, il ne vous empêchera pas de descendre dans ce Cercle cette Vallée profonde \& infecte trembla jusques dans ses fondemens, \& les secousses furent si violentes, que je crus que l'Univers alloit rentrer dans le chaos.

Precisiamo tuttavia che Moutonnet traduce molti passi ignorati in precedenza e vi apporta sostanziali modifiche che non mancano di imprimere al suo lavoro un senso opposto a quello di Détouteville. D'altra parte l'eventuale influenza della traduzione di Détouteville non appare nei passi più famosi, come l'inizio del canto XXXIII ${ }^{12}$, del quale Moutonnet ci fornisce la versione più poetica. La sua decisione di rendere al tempo presente la narrazione degli stenti di Ugolino è ciò che le conferisce maggiore liricità, differenziandola dalle altre due. Ma sceglie di risolvere l'ambiguità del famoso verso dantesco «poscia, più che 'l dolor, poté 'l digiuno»: «Enfin la faim, plus puissante que ma douleur, termina tous mes tourments» (p. 551-553). Moutonnet recalcitra di fronte a qualsiasi sfumatura di cannibalismo. Figurarsi, quindi, se poteva tradurre le oscenità dantesche... 
A loro posto, si troveranno delle perifrasi, talora prese pari pari da Détouteville: per descrivere l'inversione del capo degli indovini, costretti per contrappasso a guardare solo dietro, «le natiche bagnava per lo fesso» (XX, v. 24) era espressione troppo audace per essere tradotta in francese, così riprende dal suo predecessore: «les larmes qui couloient de leurs yeux, baignoient leurs talons». Il cortocircuito operato diventa divertente se confrontato all'originale.

Ma il suo ritegno sfocia raramente in un ritorno del represso come nella traduzione proposta da Détouteville per il v. 139 del canto XXI: «elli avea del cul fatto trombetta». Il segnale sconcio con il quale Barbariccia avverte gli altri demoni deve aver causato un sussulto in Détouteville che traduce così: «Barbariccia les contint dans le devoir» (p. 105). La censura a cui ha sottoposto il verso non impedisce all'incontinenza di Barbariccia di emergere, pur negata.

La pudicizia di Moutonnet, comunque, va anche oltre la necessità di "envelopper les ordures": per rendersene conto, basta leggere il V canto, dove l'episodio di Paolo e Francesca è reso con grande finezza poetica. Ciò non toglie che, nella nota 5 del canto V, prima di fornire la ricostruzione storica del celebre passo e di precisare l'identità dei protagonisti, Moutonnet si scagli contro i romanzi, rinnovando quella ostilità contro le opere di invenzione e di intrattenimento che aveva condotto nel 1737 alla loro proscrizione. In questa nota sembrano riecheggiare le parole del Père Porée, autore di quella requisitoria contro le autorità civili del tempo che condusse al divieto di pubblicazione dei romanzi:

Cet épisode [l'amore di Paolo e Francesca] est la critique la plus forte de la lecture des Romans, \& de nos Brochures éphémères qui gâtent l'esprit, énervent l'ame, souillent l'imagination, corrompent le coeur, \& causent les ravages les plus funestes dans la Société (p. 127).

Dunque, porre l'accento, in prima istanza, sull'intermediazione corruttrice della finzione letteraria serve a discolpare quei peccatori vittime dei loro stessi sensi: l'intensa partecipazione emotiva che suscitano le parole di Francesca deve trovare un canale di liceità. E infatti Moutonnet, preoccupato soprattutto di giustificare e legittimare la passione dei due amanti, riporta in nota l'aneddoto trasmesso dal Boccaccio invertendone i protagonisti: secondo quanto dice Moutonnet, sarebbe stato Paolo il marito destinato a Francesca. "Lanciotto" (Gianciotto) l'avrebbe quindi sposata per conto di suo fratello, usurpandone poi, per prepotenza, il diritto matrimoniale. In questo modo la loro passione viene totalmente legittimata così come la compassione di Dante. Insomma Moutonnet, sensibile alla poesia del canto V, cerca di trasmetterla senza far abdicare la morale.

\section{Fra spiritualità e materialismo}

Fin dalla prima nota Moutonnet si mostra alieno allo spirito materialista del suo tempo, affermando che solo la religione sa conciliare i due aspetti dell'animalità e della spiritualità insiti nella natura umana.

Non è un caso che il suo commento punti soprattutto sul significato anagogico del poema e la sua traduzione insista dall'inizio alla fine sulla chiave religiosa ${ }^{13}$. Ciò tutto sommato stupisce meno il lettore moderno di quanto non facciano non solo

(13) Egli, pur concedendo di sciogliere qualche allegoria, si pronuncia sulla assoluta inutilità delle spiegazioni allegoriche ("Vie de Dante" in La Divi- ne Comédie de Dante Alighieri, par Moutonnet DE Clatrfons, op. cit., pp. 45 e 37 ). 
l'aridità della traduzione di Détouteville, ma soprattutto la sua quasi totale ignoranza delle implicazioni religiose. Naturalmente Moutonnet eccede laddove si mostra incredulo che Dante voglia designare con «l'ombra di colui che fece per viltade il gran rifiuto» (III, 59-60) il papa Celestino V canonizzato dalla Chiesa (p. 93). Quanto a Rivarol, assume l'atteggiamento contrario a quello di Moutonnet, asserendo non solo che sarebbe penalizzante limitarsi all'aspetto teologico e ignorare le implicazioni e i riferimenti mitologici che Dante vi ha intrecciato ${ }^{14}$, ma arrivando persino ad affermare: «il semble qu'aujourd'hui on ne puisse plus traiter les grands sujets mystiques d'une manière sérieuse» ${ }^{15}$.

Nulla di tutto questo nella traduzione di Détouteville.

La completezza sembra essere il solo merito di questa traduzione che rivela molte debolezze rispetto sia al rigore storico e alla precisione di Rivarol che al virtuosismo stilistico di Moutonnet. Ma esiste un aspetto che merita di essere messo in evidenza. Perché se è vero che l'interesse europeo per l'Inferno è associato a una sensibilità romantica, è altrettanto indiscutibile che il rigore tassonomico con il quale Dante ha presentato l'aldilà doveva essere ben allettante per gli uomini del Settecento. Già la Commedia si presenta come un viaggio, allegorico s'intende, nell'aldilà. Dare per scontata la dimensione allegorica, tuttavia, non va da sé. Basta considerare come vengono tradotte le prime tre terzine:

\section{DÉTOUTEVILLE}

J'étois à la fleur de mes ans, lorsque je m'égarai dans une forêt obscure. Je ne voyois point de route pour en sortir. Mon embarras ne peut se décrire. L'idée seule renouvelle encore mes frayeurs: non, la mort n'est pas plus affreuse que la situation où je me trouvai; cependant elle me fut utile par les connoissances qu'elle me procura.

\section{MOUTONNET}

Au milieu de la course de nos jours je me trouvai dans une forêt obscure, où j'errois au hazard, après avoir quitté le sentier battu. Il ne me seroit pas aisé de dire quelle étoit cette forêt épaisse, pénible \& sauvage; son ressouvenir renouvelle ma frayeur: la Mort est beaucoup moins affreuse. Je parlerai d'abord des objets épouvantables que j'apperçus, \& ensuite de la rencontre heureuse que je fis.

\section{RIVAROL}

J'étais au milieu de ma course, et j'avais déjà perdu la bonne voie, lorsque je me trouvais dans une forêt obscure, dont le souvenir me trouble encore et m'épouvante.

Certes, il serait dur de dire quelle était cette forêt sauvage, profonde et ténébreuse, où j'ai tant éprouvé d'angoisses, que la mort seule me sera plus amère: mais c'est par ses âpres sentiers que je suis parvenu à de hautes connaissances, que je veux révéler, en racontant les choses dont mon oeil fut témoin.
(14) «Les longs commentaires n’ont pas éclairci les difficultés, la foule des commentateurs n'ayant vu partout que la théologie: mais ils auraient dû voir aussi la mythologie, car le poète les a mêlés». Dopo aver svolto le allegorie principali legate a Dante (animalità), Virgilio (filosofia morale), e
Beatrice (teologia) afferma che «il est difficile de se figurer qu'on puisse faire un beau poème avec de telles idées», De la vie du Dante, in Rivarol, Oeuvres Complètes, tome III, Genève, Slatkine Reprints, 1968 (Paris 1808) pp. XVII-XVIII. (15) Ibid., pp. XXIII-XXIV. 
Il confronto mette in evidenza come Détouteville privilegi il senso letterale a detrimento della polisemia della Commedia: nessuna metafora segnala l'emblema dell'umanità persa nel peccato; in compenso viene sottolineata l'utilità della conoscenza che questo viaggio ha procurato. L'avversione per il dogma cristiano che è un tratto caratteristico dell'epoca, espresso con singolare virulenza da Voltaire ${ }^{16}$, si traduce in una lingua di grado zero: le infinite variazioni stilistiche di Dante sono ridotte, appianate, normalizzate e in fondo l'Inferno è descritto, con piglio enciclopedico, come un ulteriore regno della natura.

La precisione della descrizione induce Détouteville a utilizzare forme sintatticamente consequenziali, che razionalizzano all'estremo. Si prenda come esempio il canto XIII e la descrizione degli scialacquatori inseguiti dalle cagne infernali:

In quel che s'appiattò miser li denti,

e quel dilaceraro a brano a brano;

poi sen portar quelle membra dolenti (XIII, 127-129).

Come sempre Dante è essenziale, ed è nella lentezza del v. 128 nonché nella permanenza del dolore in quei corpi ormai dilaniati che risiede l'orrore della pena. Détouteville ignora tutto questo e traduce semplicemente il fatto, insistendo tramite l'incidentale sulla consequenzialità dei fatti che risulta alla fin fine più importante di qualsiasi reazione emotiva:

Elles se mirent à mordre celui qui étoit caché dans le buisson; et, l'ayant mis en pièces, elles emportèrent ses membres ensanglantés.

Molto diversa la resa di Moutonnet, che non esita a dilungarsi per enfatizzare l'orrore della terzina:

elles se jettent sur l'Ombre couchée dans les épines, la déchirent, \& emportent avec leurs dents ses membres palpitans \& encore sensibles à la douleur.

Quanto a Rivarol, la sua traduzione si situa proprio a metà strada fra l'arido resoconto dei fatti e l'intensità del sentire, in quanto utilizza la stessa struttura sintattica di Détouteville ma con un vocabolario più vicino a Moutonnet:

elles se jetèrent en fureur sur celui qui haletait dans le buisson; et l'ayant déchiré entre elles, en emportèrent les membres palpitants.

È molto frequente che, in Détouteville, la ricerca di precisione vada a discapito della tensione emotiva del testo, per questo riesce a tradurre meglio quei canti, come il XXV, in cui la minuziosità della descrizione di Dante copre l'orrore dell'immagine. $\mathrm{Ma}$ arriva persino ad aggiungere elementi che rafforzano la già precisa geometria dantesca: nel canto XVIII, per esempio, vi è la descrizione delle Malebolge:

Luogo è in inferno detto Malebolge,

tutto di pietra di color ferrigno,

come la cerchia che dintorno volge.

Nel dritto mezzo del campo maligno

vaneggia un pozzo assai largo e profondo,

di cui suo loco dicerò l'ordigno.

(16) Che non apprezzava affatto Dante. Si veda A. FarinelLI, op. cit., pp. 249-251. 
Quel cinghio che rimane adunque è tondo

tra 'l pozzo e 'l pié de l'alta ripa dura,

e ha distinto in dieci valli il fondo.

Il rinvio a tempo debito della descrizione del cerchio dovette sembrare inopportuno a Détouteville che vi inserisce un'aggiunta esplicativa ma inevitabilmente analitica e monotona:

Cette roche taillée perpendiculairement formoit les murailles du huitième cercle, nommé malboge, dans lequel sont renfermés dix autres cercles, qui depuis la circonférence jusqu'au centre vont toujours en diminuant. Le premier est habité par les séducteurs; le second, par les flatteurs; le troisième, par les simoniaques; le quatrième, par les devins; le cinquième, par les usuriers; le sixième, par les hypocrites; le septième, par les larrons; le huitième, par ceux qui s'ingèrent à donner de mauvais conseils; le neuvième, par les auteurs du scandale; et le dixième, par les faussaires.

Sappiamo che, laddove si guardi al testo di Dante nel suo rapporto con la realtà, esso postula come reale proprio l'aldilà che è il frutto della sua immaginazione ${ }^{17}$. $\mathrm{Pa}$ radossalmente, la traduzione piatta, banale, particolareggiata di Détouteville sembra proprio sortire il medesimo effetto: con intento tassonomico, essa si pone a completamento di una descrizione del reale alla quale mirava il sapere enciclopedico dell'epoca. In questo sta la straordinaria, e del tutto involontaria, portata delle traduzioni settecentesche della Divina Commedia, e soprattutto di quella di Détouteville, di un inesorabile scialbore, ma proprio in ciò preparatoria alla romantica considerazione dell'aldilà come una dimensione necessaria alla vita dell'uomo.

Ciò appare ancora più evidente nei passaggi più "filosofici":

\section{DÉTOUTEVILLE}

Mon fils, gravez dans votre coeur les vérités que je vais vous annoncer. De même que le créateur a formé les cieux, et y plaça le soleil pour éclairer toutes les parties du monde en distribuant également sa lumière; de même il a établi la fortune sur la terre, pour faire passer successivement les biens temporels d'une famille à l'autre, malgré les soins que se donnent les hommes pour les conserver. C'est par ses ordres seuls, qu'une partie des humains commande, et que l'autre obéit. (p. 34)

\section{MOUTONNET}

O mon Fils, prêtez une oreille attentive à mes discours, \& gravez-les au fond de votre coeur! Celui dont la suprême sagesse embrasse tout, a créé les Etoiles, \& leur a donné des intelligences pour les conduire, afin que la lumière fut également réfléchie \& distribuée dans tout l'Univers; il a de même accordé à la Fortune un pouvoir général sur les biens, \& sur les grandeurs humaines. Elle les régit \& les gouverne avec un empire absolu. Malgré tous les raisonnemens des hommes, elle fait passer à son gré la puissance d'un peuple dans des mains étrangères: elle dépouille les uns, enrichit les autres, distribue les sceptres \& les chaînes;

(pp. 154-155)

\section{RIVAROL}

Ecoute-moi, et que ma parole descende dans ton coeur... Celui dont le regard embrasse les mondes, entrelaçant jadis leurs orbes dans les cieux, dit à ses ministres de régler la course des torrents de lumière, et l'harmonie des globes. A sa voix, une divinité puissante vint ici-bas s'asseoir au trône des splendeurs mondaines. C'est elle dont la main promène de peuple en peuple et de race en race la honte ou la gloire, et qui trouble à son gré les conseils de l'humaine sagesse.

(pp. 57-58) 
Le note di cui Moutonnet correda questo passo tendono a conciliare la visione dantesca con le Sacre Scritture: «Le Poëte appelle Dieux, les Intelligences qui président à chaque Planète; ce n'est point une hérésie, c'est dans le sens de l'Ecriture sainte, lorsqu'elle dit, en parlant des hommes: Dii E filii excelsi omnes» (p. 160). Anche Rivarol puntualizza, nella nota 6, che «L'église admet ce système» (p. 61).

Ma la versione di Rivarol possiede uno spessore metaforico che relega immediatamente la creazione in una dimensione fantastica e poetica, quasi favolistica. Moutonnet insiste, come anche in altri passi, sulla saggezza nonché sulla infinita supremazia del creatore che il raziocinio umano non arriva a comprendere. Per Détouteville invece, il creatore è semplicemente colui che ha plasmato i cieli e la terra, senza nessun bisogno di fioritura metaforica, né di spessore spirituale. Come si vede, Rivarol concilia le due versioni e, scegliendo il mito, si situa a metà strada fra il materialismo dell'una e la religiosità dell'altra.

L'intenzione di Rivarol di dare ampio spazio all'aspetto descrittivo si precisa in un paragone insolito, che a prima vista sorprende. Nella nota 5 dell'undicesimo canto, infatti, il traduttore pronuncia un apprezzamento dell'Inferno dantesco e, riconoscendolo come ben superiore alla filosofia scolastica del suo tempo, lo paragona all'Esprit des lois di Montesquieu:

Ses distinctions sont nettes, et sa théologie fort simple. Le début de l'esprit des lois est le même quant au sens. Au livre 26, chap. I, Montesquieu parle de cette sagesse humaine qui a fondé toutes les sociétés. Il l'appèle droit politique général, et dit que c'est la sublimité de la raison humaine, que de statuer l'ordre et les principes qui doivent gouverner les hommes.

Questa affinità viene desunta dalla parentela che Rivarol riscontra fra i peccati descritti da Dante e i crimini individuati da Montesquieu, similarità espressa a chiare lettere nell'introduzione: «Il n'est point de crime qui soit oublié dans la distribution des supplices que le poète rencontre d'un cercle à l'autre: souvent une enceinte est partagée en différents donjons; mais toujours avec une telle suite dans la gradation des crimes et des peines, que Montesquieu n'a pas trouvé d'autres divisions pour son esprit des lois» ${ }^{18}$. Peraltro il paragone di Rivarol si riflette inevitabilmente su scelte traduttive che rendono conto di un'interpretazione a dir poco tendenziosa, come in questo esempio:

«Filosofia», mi disse, «a chi la 'ntende,

nota, non pure in una sola parte,

come natura lo suo corso prende

dal divino 'ntelletto e da sua arte;

e se tu ben la tua Fisica note,

tu troverai, non dopo molte carte,

che l'arte vostra quella, quanto pote,

segue, come 'l maestro fa 'l discente;

sì che vostr'arte a Dio quasi è nepote (XI, 97-105).

\section{Così traduce Rivarol:}

Ecoute, donc, reprit-il, ce que la philosophie crie sans cesse: La nature découle de l'essence de Dieu même qui lui donna des lois. Or, si tu suis les maximes de cette philosophie, tu reconnaîtras que les lois humaines empruntent leur faible éclat de ces lois éternelles du monde, et que l'homme a été le disciple de son Dieu. Ainsi par le droit de son origine la sagesse de l'homme, seconde fille du ciel, ira s'asseoir entre la nature et son auteur. 
In questo canto in cui l'ordine e l'efficacia dell'esposizione fanno sì che venga abbandonata la poesia per un tono più didattico, il pensiero di Dante concerne il lavoro umano derivante dalla natura che a sua volta imita Dio. Dunque laddove il poeta si riferisce alle leggi del lavoro umano (arte), Rivarol sposta il discorso all'ambito giuridico e intende con "arte vostra" la saggezza. Esiste dunque un rapporto fra questa forzatura interpretativa e il paragone con Montesquieu.

Ma la forzatura imprime una direzione alla traduzione, anche nel caso di Détouteville, che traduce così questo stesso passo:

La philosophie, me répondit-il, vous apprend que la nature entière prend sa source dans l'intelligence divine. La physique, que vous aimez tant, a dû vous démontrer que toute notre science dépend de cette intelligence, et la suit autant qu'elle peut, comme l'écolier suit son maître. Ainsi c'est de Dieu seul que nous pouvons recevoir la science.

Per Détouteville «l'arte vostra» dantesca, cioè l'attività, il lavoro umano, era «notre science», il nostro sapere, laddove per Rivarol diverranno le «lois humaines».

Questa trasposizione dell'ordinamento divino a livello umano è evidente anche in altri passi, come nel secondo canto in cui Virgilio replica a Beatrice e comincia apostrofandola così:

O donna di virtù sola per cui

l'umana spezie eccede ogne contento

di quel ciel c'ha minor le cerchi sui (II, 76-78).

Si mettano a confronto le tre traduzioni:

\section{DÉTOUTEVILLE}

modèle de vertu! vous, par qui l'espèce humaine est audessus de tout ce qui respire,

\section{MOUTONNET}

O Femme ornée de toutes les Vertus, me suis-je écrié, vous qui donnez seule la connoissance sublime qui élève l'esprit humain au dessus des choses terrestres

\section{RIVAROL}

O femme, qui brûlez de ce feu divin, par qui seul la race de l'homme a mérité l'empire de son séjour!

Sappiamo che Beatrice rappresenta la Teologia e ciò è implicito in ciascuna delle versioni, anche se Moutonnet e Rivarol vi aggiungono una nota esplicativa. La differenza appare nella traduzione del verso "l'umana spezie eccede ogne contento": Détouteville non lascia spazio al trascendente; Moutonnet al contrario preferisce riferirsi all' "esprit" e connota la superiorità umana di un senso intellettualistico oltre che spirituale. Rivarol implica nella sua traduzione tanto la divinità, a cui fa esplicito riferimento, che una superiorità morale tradotta in termini politici. La necessità di mostrare con precisione l'ordinamento dell'inferno è espressa anche in quel capitolo dell'introduzione intitolato "De l'état des morts", in cui spiega la differenza nelle diverse culture fra "âme, corps et ombre" concludendo con questa frase:

Toutes ces distinctions, que j'ai tâché d'établir avec quelque clarté, sont un peu confuses chez les anciens: ce sont bien des notions différentes, mais dont les limites ne sont pas bien marquées. Il y a dans la fable autant de législateurs que de poètes, et il ne faut pas donner un code à l'imagination. 
La traduzione di Rivarol ha fatto tesoro delle potenzialità dei suoi predecessori nella misura in cui l'attenzione alla spiritualità e alla dimensione fantastica si è affiancata alla descrizione della "natura" dell'aldilà, come mostra peraltro la sua assimilazione all'Esprit des Lois di Montesquieu.

In questo forse sta la ragione del suo successo. Situato a cavallo fra due epoche, Rivarol seppe cogliere l'essenziale dell'una e dell'altra, accontentando il gusto di un pubblico ancora debitore del materialismo settecentesco, ma già proiettato verso la spiritualità del secolo successivo.

Grazie al rigore quasi tassonomico di Détouteville che, combinato alla dimensione allegorico-spirituale di Moutonnet è sfociato nella misurata combinazione di mistero e di ordine della traduzione di Rivarol, l'Inferno sembrava pronto, alla fine del Settecento, ad essere considerato anche in Francia la descrizione dell'inferno quale lo è nella nostra cultura, l'immagine mitica che ne abbiamo ormai indelebilmente impressa nella memoria.

Non sarà impossibile provare ciò attraverso un indizio che permette di mostrare come le divergenze fra le traduzioni settecentesche della Divina Commedia siano il segno di un'evoluzione significativa: basta infatti porre attenzione ai nomi propri, non dei peccatori evidentemente, ma dei diavoli e dei luoghi infernali. Come sappiamo, Dante elenca i demòni nel canto XXI e i loro nomi, come ci si può ragionevolmente aspettare, possiedono una sonorità italiana. $\mathrm{Ma}$, a meno che non si consideri che essi provengano dall'Italia, sarebbe naturale tradurne i nomi, così come esistono, in francese, gli "archanges" Gabriel, Michel e tutti gli altri angeli (che, va riconosciuto, esistevano prima di Dante e al di fuori della Divina Commedia). Ebbene, i nomi dei diavoli restano invariati nella traduzione di Moutonnet: il suo rigore filologico lo spinge a riportarli esattamente. Anche Détouteville li riporta, ma francesizza Alichino in Aliquin e si mostra meno attento all'esatta grafia degli altri (così Libicocco diventa Libicoco, Draghignazzo si contrae in Draghinzo e Graffiacane si muta in Graffiacone). Allo stesso modo, le Malebolge diventano per lui "Malboge". Tutti nomi, se non infranciosati, quanto meno adattati alla pronuncia francese. Insomma, i diavoli cominciano a non essere più solo italiani...

La scelta di Rivarol è ancora diversa: molto intelligentemente, egli evita di nominare i demòni e li designa tutti genericamente: «Alors, il les appela par leurs noms» (p. 170). È vero che ciò risponde a un preciso intento stilistico. Se non li nomina, infatti, è per non ridicolizzarli, come fa Dante, che rendendoli risibili e sconci vuole diminuirne la potenza e metterne in luce l'indegnità: i suoi sono spaventosi e non grotteschi. Giustificando la sua scelta nella terza nota del canto XXII, Rivarol afferma di aver «négligé de rendre les noms que le Dante a donné à ces dix démons, parce qu'ils sont d'une harmonie ridicule» (p. 182) e la sua traduzione vuole mantenere il testo al livello elevato. Il risultato che ottiene travalica comunque la classica ripartizione degli stili poiché, se da una parte accentua la dimensione medievale del testo, come imponeva la svolta della sensibilità in atto di compiersi, dall'altra rimane ancorato a quello spirito classicista che si risolve in una assimilazione dell'opera alla cultura francese. Scegliere lo stile sublime non aveva dunque implicato, per Rivarol, l'evacuazione di quegli aspetti riconducibili al materialismo.

Dopo che Détouteville aveva normalizzato il fantastico, dopo che Moutonnet l'aveva arricchito della sua originale dimensione spirituale, la Divina Commedia sarebbe stata pronta ad assumere nel patrimonio culturale francese quella statura mitica che già possedeva in Italia. Bisognerà tuttavia attendere l'epoca romantica per vedere esaltati gli aspetti medievali dell'opera, in ciò cogliendo e sviluppando appieno quanto i traduttori settecenteschi, per esigenze di rispetto dei canoni di bellezza ancora in auge, avevano relegato in margine. 